\section{Quelques théorèmes de nature tauberienne}

par

J. KARAMATA (Beograd).

Le présent travail fait en quelque sorte suite à ma Note parue dans les Studia Mathematica ${ }^{1}$ ). L'objet en est de donner quelques applications immédiates du théorème général 5, démontré dans la dite Note, dont l'énoncé est le suivant

Thé or èm e A. Soit $\alpha_{n}(x)$, pour $0 \leqslant x \leqslant 1, n=1,2,3, \ldots$, une suite de fonctions satisfaisant aux conditions suivantes:

$$
\begin{gathered}
\alpha_{n}(1)=0, \text { pour tout } n=1,2,3, \ldots, \\
\liminf _{n=\infty} \min _{x<t \leqslant x^{\lambda}}\left\{\alpha_{n}(t)-\alpha_{n}(x)\right\}>-w(\lambda) \rightarrow 0 \quad(1>\lambda \rightarrow 1)
\end{gathered}
$$

pour tout $0<x<1$, et
$\int_{0}^{1} t^{k} d\left\{\alpha_{n}(t)\right\} \rightarrow \int_{0}^{1} t^{k} d\{\alpha(t)\} \quad(n \rightarrow \infty)$, pour tout $k=1,2,3, \ldots ;$ alors $\alpha_{n}(x) \rightarrow \alpha(x)(n \rightarrow \infty)$ en tout point de continuite $x$ de $\alpha(x)$.

En particulier, nous donnerons une réeponse à une question proposée par M. A. ZYGMund, qui consiste à savoir à quelles conditions doit-on assujetir la suite de nombres $s_{v}(\nu=0,1,2,3, \ldots)$ pour qu'on puisse de la relation

$$
(1-r) \sum_{\nu=0}^{n} s_{\nu} r^{\nu}=s+o\left\{(1-r)^{h}\right\} \quad(r \rightarrow 1) \quad(h \geqslant 0)
$$

conclure que

$$
\frac{1}{n} \sum_{\nu=0}^{n} s_{v}=s+o\left\{n^{-h}\right\} \quad(n \rightarrow \infty)
$$

1) J. Ka ramata, Sur le rapport entre les convergences d'une suite de fonctions et de leurs moments avec application à l'inversion des procédés de sommabilité, Studia Mathematica 3 (1931) p. 68-76.
Pour pouvoir déduire du théorème $\mathrm{A}$, d'une manière plus directe, les théorèmes de nature tauberienne, nous lui donnerons d'abord une autre forme, d'ailleurs équivalente. Ainsi, en remplaçant dans les fonctions et intégrales y figurant $t$ par $e^{-t}$, $\alpha_{n}\left(e^{-t}\right)$ par $A_{n}(t)$, et $y$ faisant quelques autres légères modifications, l'on obtient le

Théo rè me B. Soit $A_{n}(x)(n=1,2,3, \ldots)$ une suite de fonctions définies dans $(0, \infty)$, telles que les intégrales

existent, et

$$
\int_{i}^{\infty} e^{-k t} d\left\{A_{n}(t)\right\} \quad(n, k=1,2,3, \ldots)
$$

$$
\text { (1) } \quad A_{n}(0)=0 \text {, pour tout } n=1,2,3, \ldots
$$

Si ces fonctions satisfont à la condition

$$
\text { (2) } \quad \lim _{n=\infty} \inf \min _{x<t<\lambda x}\left\{A_{n}(t)-A_{n}(x)\right\}>-w(\lambda) \rightarrow 0
$$

lorsque $1<\lambda \rightarrow 1$, pour tout $x$, alors des relations

$$
\int_{0}^{\infty} e^{-k t} d\left\{A_{n}(t)\right\} \rightarrow \int_{0}^{\infty} e^{-k t} d\{A(t)\} \quad(n \rightarrow \infty),
$$

pour tout $k=1,2,3, \ldots$, il résulte que

(4) $A_{n}(x) \rightarrow A(x) \quad(n \rightarrow \infty)$ en tout point de continuité $x$ de $A(x)$. L'indice $n$ figurant dans ce théoréme peut de même varier d'une manière continue de 0 à $\infty$, ce qui se présentera, du reste, dans les applications suivantes.

Appliquons, à présent, le théorème $\mathrm{B}$ à l'étude de la valeur asymptotique d'une fonction $A(x)$ qui satisfait à la relation suivante :

$$
\int_{0}^{\infty} e^{-v t} d\{\mathrm{~A}(t)\}=o\left\{\varphi\left(\frac{1}{\sigma}\right)\right\}(\sigma \rightarrow 0)
$$

$\varphi(x)$ étant une fonction positive telle que

(6) $\quad \varphi(u x)=O\{\varphi(x)\}(x \rightarrow \infty)$, pour tout $u>0$.

A cet effet, posons dans (5) $\sigma=k / n, k>0$, puis remplaçons $t / n$ par $t$; l'on en déduit alors la relation

$$
\int_{0}^{\infty} e^{--k t} d\{A(n t)\}=o\{p(n \mid k)\} \quad(n \rightarrow \infty),
$$


valable pour tout $k>0$. En divisant cette relation par $\varphi(n)$ et en tenant compte de (6), l'on obtient:

$$
\int_{0}^{\infty} e^{-k t} d\left\{\frac{A(n t)}{\varphi(n)}\right\} \rightarrow 0(n \rightarrow \infty), \text { pour tout } k>0 .
$$

En posant donc

$$
A_{n}(t)=\frac{A(n t)}{\varphi(n)}
$$

et en appliquant à cette fonction le théorème $\mathrm{B}$, on en déduit le théorème suivant:

Thé o r è me 1. Soit $\varphi(x)$ une fonction positive et telle que ((6)) de la relation

$$
\varphi(u x)=O\{\varphi(x)\}(x \rightarrow \infty), \text { pour tout } u>0 ;
$$

$$
\int_{0}^{\infty} e^{-\sigma t} d\{A(t)\}=o\left\{\varphi\left(\frac{1}{\sigma}\right)\right\}(\sigma \rightarrow 0)
$$

il résulte

$$
\text { (7) } \quad A(x)=o\{\varphi(x)\}(x \rightarrow \infty)
$$

toutes les fois que l'on a

(8) $\quad \liminf _{x=\infty} \min _{x<x^{\prime}<\lambda_{x}}\left\{\frac{A\left(x^{\prime}\right)-A(x)}{\varphi(x)}\right\}>-w(\lambda) \rightarrow 0$

lorsque $1<\lambda \rightarrow 1$.

En ce qui concerne la condition (8), on peut la remplacer, dans le cas où $A(x)$ possède une dérivée première, par une plus simple, mais moins générale:

Théor èm e 2. Si la fonction $\varphi(x)$ satisfait da la condition (6), de la relation (5) il résultera (7) toutes les fois que $A(x)$ possède une dérivée première telle que

$$
A^{\prime}(x)>O\{\varphi(x) / x\}(x \rightarrow \infty) .
$$

On peut, en effet, montrer que la condition (8) est satisfaite toutes les fois que (9) est rempli. Car, en intégrant cette dernière inégalité entres les limites $x$ et $x^{\prime} \leqslant \lambda x, \lambda>1$, l'on en déduit, en tenant compte de (6),

$A\left(x^{\prime}\right)-A(x)>-M \int_{x}^{x^{\prime}} \varphi(t) d t \mid t>-M \int_{1}^{\lambda} \varphi(x t) d t / t>-M^{\prime} \varphi(x) \lg \lambda \rightarrow 0$

avec $\lambda \rightarrow 1$, ce qui montre bien que la condition (8) est satisfaite dès que (9) l'est.

En posant, à présent, dans les théorèmes 1 et 2

l'on en déduit le

$$
A^{\prime}(x)=s(x)-s \text { et } \psi(x)=\varphi(x) / x,
$$

$\mathrm{T} \mathrm{h}$ é or è $\mathrm{m} \mathrm{e} 3$. De la relation

$$
\sigma \int_{0}^{\infty} e^{-\sigma t} s(t) d t=s+o\left\{\psi\left(\frac{1}{\sigma}\right)\right\} \quad(\sigma \rightarrow 0)
$$

il résulte que

$$
\frac{1}{x} \int_{0}^{x} s(t) d t=s+o\{\psi(x)\}(x \rightarrow \infty)
$$

si la fonction $s(x)$ satisfait à l'une des deux conditions suivantes:

$$
\begin{gathered}
\liminf _{x=\infty} \min _{x<x^{\prime}<\lambda x} \frac{1}{x} \int_{x}^{x^{\prime}} s(t) d t \geqslant s(\lambda-1)-w(\lambda) \psi(x) \\
(w(\lambda) \rightarrow 0,1<\lambda \rightarrow 1),
\end{gathered}
$$

où bien

(13)

$s(x) \geqslant s-M \psi(x)(M>0)$, pour tout $x$, $\psi(x)$ satisfaisant $\grave{a}$ la condition que

$$
\psi(u x)=O\{\psi(x)\}(x \rightarrow \infty), \text { pour tout } u>0 .
$$

En particulier, si l'on pose dans ce théorème

$$
s(x)=s_{n} \text { lorsque } n \leqslant x<n+1 \quad(n=0,1,2, \ldots)
$$

et $e^{-u}=r$, l'on en déduit que de la relation

$$
(1-r) \sum_{v=0}^{\infty} s_{p} r^{v}=s+o\left\{\psi\left(\frac{1}{1-r}\right)\right\}(r \rightarrow 1)
$$

il résultera

$$
\frac{1}{n+1} \sum_{\nu=0}^{n} s_{v_{v}}=s+o\{\psi(n)\}(n \rightarrow \infty)
$$

toutes les fois que la suite $s_{n}$ satisfait à la condition

$$
s_{n} \geqslant s-M \psi(n) \text {, pour tout } n=0,1,2, \ldots(M>0),
$$

$\psi(x)$ étant une fonction positive satisfaisant à la condition (14).

Si l'on pose dans ce dernier résultat $\psi(x)=x^{-h}(h \geqslant 0)$, l'on obtient une réponse à la question posée au début de cette Note.

(Reģu par la Rédaction le 8. 6. 1932). 